\title{
A Computational Model for Pattern and Tile Designs Classification Using Plane Symmetry Groups
}

\author{
José M. Valiente ${ }^{1}$, Francisco Albert² ${ }^{2}$ and José María Gomis ${ }^{2}$ \\ ${ }^{1}$ DISCA \\ ${ }^{2}$ DEGI , Universidad Politécnica de Valencia, Camino de Vera s/n, \\ 46022 Valencia, Spain \\ jvalient@disca.upv.es, \{fraalgi1,jmgomis\}@degi.upv.es
}

\begin{abstract}
This paper presents a computational model for pattern analysis and classification using symmetry group theory. The model was designed to be part of an integrated management system for pattern design cataloguing and retrieval in the textile and tile industries. While another reference model [6], uses intensive image processing operations, our model is oriented to the use of graphic entities. The model starts by detecting the objects present in the initial digitized image. These objects are then transformed into Bezier curves and grouped to form motifs. The objects and motifs are compared and their symmetries are computed. Motif repetition in the pattern provides the fundamental parallelogram, the deflexion axes and rotation centres that allow us to classify the pattern according its plane symmetry group. This paper summarizes the results obtained from processing 22 pattern designs from Islamic mosaics in the Alcazar of Seville.
\end{abstract}

\section{Introduction}

The interest of plane symmetry group theory for the design and cataloguing of regular plane segmentations can be seen in works such as [1] or [2]. These works analyze, with mathematical and geometrical rigor, the design patterns used by the ancient Islamic handcraft workers for covering architectural surfaces and walls. In addition, these works have become a key reference for most contributions that, in the form of computer models have analyzed their pattern geometries in recent years. Most of these research works describe design pattern geometry and provide tools ,like Shape Grammars [14], for design pattern generation which are very useful in the world of Computer Graphics. However few of such works analyze pattern designs using computer vision. Nevertheless, from this perspective, there are many works on the analysis of independent symmetries, [3] [4] [5], although few works have studied symmetry groups in images. Among these works, it is worth mentioning the theoretical approaches of [4] and [5], and particularly the computational model proposed by Y. Liu, R.T. Collins and Y. Tsin [6]. This, as opposed to the model presented in this paper, works in image space and thus obtains global symmetries, with no specification of pattern objects and motifs. We have taken it as a reference model for our work. 
In this paper we propose an alternative computational model which, based on symmetry group theory [1], [2], allows the automatic analysis, decomposition and classification of the digital image of a regular design pattern. To evaluate this model's capacity to analyze historical Islamic design patterns, the authors analyze the tile patterns used in one of the most emblematic Islamic buildings in Spain: the Alcazar of Seville, built between 1364 and 1366. This palace possesses one of the largest and most beautiful patterns in Islamic Art.

\section{Design Patterns and Tile Designs: Identification of PSG}

Design patterns and tile designs are both the result of a systematic repetition of one given geometrical form. However, each has certain inherent characteristics [3]: in the case of a design pattern, the repeated geometrical form has no constraints, since the result is a set of independent geometrical forms more or less close to each other. In the case of tile designs, the repeated form necessarily requires a given shape to avoid gaps or overlapping. "Geometrical Form" here means what is perceived or seen, and comprises any figure, image or drawing used as unit motif to create a pattern design.

Despite these formal differences between design patterns and tile designs, their classification in terms of compositive syntax, is similar and in accordance with symmetry group theory. This theory states that any $2 \mathrm{D}$ pattern can be classified according to the set of geometrical transformations that transforms it into itself. Transformations that preserve distances are known as isometries, and the plane isometries are: rotations, translations, reflections and glide reflections. The set of isometric transformations that makes a motif coincide with itself is known as symmetry group. Three types or categories of symmetry groups are defined:

- $\quad$ Point symmetry groups (psg): including cyclic and dihedral symmetry groups. The cyclic group $\boldsymbol{C}_{\boldsymbol{n}}$ has only $\mathrm{n}$-fold rotational symmetry around the center. The dihedral group $\boldsymbol{D}_{\boldsymbol{n}}$ also includes $n$ reflection axes.

- Frieze symmetry groups (FSG): containing only one translational symmetry and other symmetries.

- Plane symmetry groups (PSG) or Wallpaper groups: containing two translational symmetries and other symmetries.

The importance of this theory lies in the fact that all design patterns and tile designs can be classified according to the FSG or PSG to which they belong. It is known that there are geometric restrictions, called 'crystallographic constraints', which limit the number of possible rotations that can be applied to completely fill the plane or frieze [7]. Accordingly, the PSG and FSG are limited to 17 and 7 classes respectively. We only address the problem of PSG identification.

In a previous work [8] we studied the specific aspects used to identify the PSG. Following other works in the literature, we suggested that the basic information on the pattern structure resides in three features:

- Fundamental Parallelogram (FP): the smallest part of the pattern that by replicating and displacing is able to generate the whole pattern. The FP is defined by two displacement vectors, the parallelogram sides, which can be used to locate the centre position of all motifs in the pattern. In [6] the FP is known as the unit lattice. 
- Design symmetries axes (DSA): the reflection or glide reflection symmetry axes of motifs present in the pattern.

- Design rotation centers $(\boldsymbol{D R C})$ : the points around the motifs can rotate to find another repetition of themselves in the pattern. According with the crystallographic constraints above mentioned, there are a limited number of possible rotations.. The corresponding DRC or $n$-fold rotation centers are featured by an order $n=2,3,4$ and 6 which indicate rotations of $180^{\circ}, 120^{\circ}, 90^{\circ}$ and $60^{\circ}$ respectively.

Thus, we propose using these three features to identify the PSG of a given pattern. Table 1 shows the strict relation between these structural descriptors and each of the 17 PSG. The first column shows standard PSG nomenclature.

Table 1. PSG classification using FP, DSA, and DRC features

\begin{tabular}{|c|c|c|c|c|c|}
\hline PSG & FP & DSA & DRC & DSA and & ith respect to FP \\
\hline P1 & S,RE, ERO,RO, P & None & \multirow{4}{*}{ None } & & \multirow[b]{2}{*}{ PM } \\
\hline PM & S,RE & RA II FP side & & $\mathrm{P} 1 \angle$ & \\
\hline PG & S,RE & GRA II FP side & & \multirow[b]{2}{*}{$P G$} & \multirow{2}{*}{$\mathrm{CM}$} \\
\hline $\mathrm{CM}$ & S,RO,ERO & RA \| FP diag & & & \\
\hline P2 & S,RE, ERO,RO, P & None & \multirow{5}{*}{$\diamond^{2 \text {-fold }\left(180^{\circ}\right)}$} & & \multirow[b]{4}{*}{$\mathrm{PGG} \perp 0 \perp$} \\
\hline PMM & $\mathrm{S}, \mathrm{RE}$ & RA II FP sides & & P2 & \\
\hline PMG & S,RE & $\begin{array}{l}\text { GRA II FP side } \\
\text { RA } \| 2^{\text {nd }} \text { FP side }\end{array}$ & & & \\
\hline PGG & S,RE & GRA II FP sides & & PMG & \\
\hline CMM & $\mathrm{S}, \mathrm{RO}, \mathrm{ROE}$ & $\begin{array}{l}\text { RA II FP diag. } \\
\text { RA II FP } 2^{\text {nd }} \text { diag. }\end{array}$ & & $\mathrm{CMM}$ & \\
\hline P3 & ERO & None & \multirow{3}{*}{$\triangle^{3 \text {-fold }\left(120^{\circ}\right)}$} & & \multirow{3}{*}{ P31M $\triangle \triangle \triangle \triangle$} \\
\hline P31M & ERO & $\begin{array}{l}\text { RA II FP sides } \\
\text { RA I| FP diag. }\end{array}$ & & $\mathrm{P} 3 \triangle$ & \\
\hline P3M1 & ERO & $\begin{array}{l}\text { RA I_ FP sides } \\
\text { RA II FP diag. }\end{array}$ & & $\mathrm{P} 3 \mathrm{M} 1$ & \\
\hline P4 & $\mathrm{S}$ & None & \multirow{3}{*}{$\square^{4-\text { fold }\left(90^{\circ}\right)}$} & & \multirow{3}{*}{$\mathrm{P} 4 \mathrm{M} \leftarrow \forall$} \\
\hline P4M & $\mathrm{S}$ & $\begin{array}{l}\text { RA \| FP sides } \\
\text { RA I| FP diags }\end{array}$ & & $\mathrm{P} 4 \square$ & \\
\hline P4G & $\mathrm{S}$ & $\begin{array}{l}\text { GRA \| FP sides } \\
\text { RA I| FP diags }\end{array}$ & & P4G 4 Q & \\
\hline P6 & ERO & None & \multirow{2}{*}{$\begin{array}{l}\text { 6-fold }\left(60^{\circ}\right) \\
\square_{\text {3-fold }\left(120^{\circ}\right)} \\
\text { 2-fold }\left(180^{\circ}\right)\end{array}$} & & \multirow[b]{2}{*}{$\mathrm{P} 6 \mathrm{M} \& \mathrm{E}$} \\
\hline P6M & ERO & $\begin{array}{l}\text { RA II FP sides } \\
\text { RA II FP diags } \\
\text { RA_I_FP sides }\end{array}$ & & P6 & \\
\hline \multicolumn{4}{|c|}{$\begin{array}{c}\mathrm{FP}=\text { parallelogram }(\mathrm{P}) \text {, square }(\mathrm{S}) \text {, rhombus }(\mathrm{RO}) \text {, rectangle }(\mathrm{RE}) \text { and } \\
\text { equilateral rhombus (ERO) }\end{array}$} & \multicolumn{2}{|c|}{$\begin{array}{c}\text { DSA }=\text { reflection axe (RA), glide reflection axe } \\
(\text { GRA) }\end{array}$} \\
\hline
\end{tabular}

\section{A Reference Computational Model}

As mentioned above, Y. Liu, R.T. Collins and Y. Tsin have recently proposed a computational model (afterwards LCT Model) for periodic pattern perception based on crystallographic group theory [6]. LCT Model input is the image containing 1D or 2D 
periodic pattern. LCT outputs are the frieze or wallpaper group the image belongs to and its median tile. Figure 1 shows a scheme of the main LCT components.

The model has four main stages: (i) Lattice detection, which is the extraction of two linearly independent vectors that describe the translational symmetry of the pattern. (ii) Median tile, which is a representative tile extracted using the median of pixels in all tile-shaped regions formed by the lattice. (iii) Test symmetries, which extract the rotation and reflection symmetries in the pattern. (iv) Classification, which classifies the pattern in one of the 17 wallpaper or 7 frieze symmetry groups.

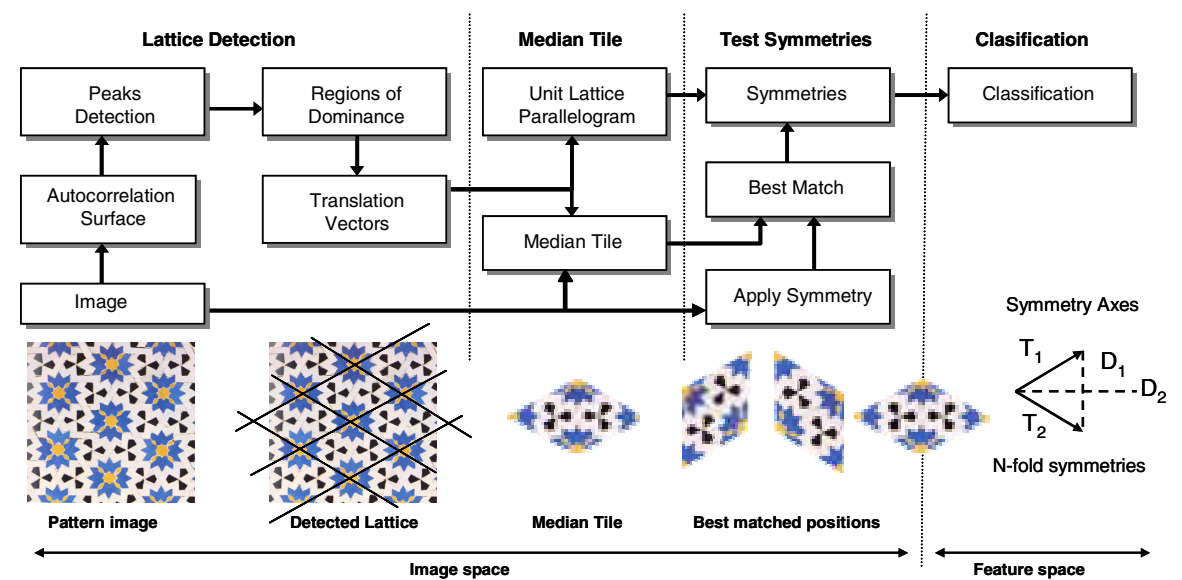

Fig. 1. A schematic workflow of the computational model reported in [6]

To prove this computational model, several synthetic and real-world pattern samples were used. The problems arise from two main causes. Firstly, real-world patterns are very noisy so they depart from ideal frieze or wallpaper patterns. Secondly, symmetry groups have hierarchical relationships among themselves, so they are not mutually exclusive classes. That means a given pattern can be classified in several symmetry groups. To address these problems, the authors propose a modified version of their computational model that uses a measurement of symmetry group distances and Geometric AIC (Akaike Information Criterion) [13]. The result is a very robust and successful algorithm only limited by practical issues, such as the use of distorted or damaged samples from the real world.

It is significant that most of the task proposed by the model is performed in the image space using pixel values. Only in the last stage are feature vectors (symmetry scores or group distances) used to classify the pattern. All the other stages require an intensive use of bitmap manipulations, with the subsequent computational requirements.

\section{The Proposed Computational Model}

We propose an alternative to the LCT model that, with the same aim and scope, attempts to approach the problem from the point of view of the graphic world, rather 


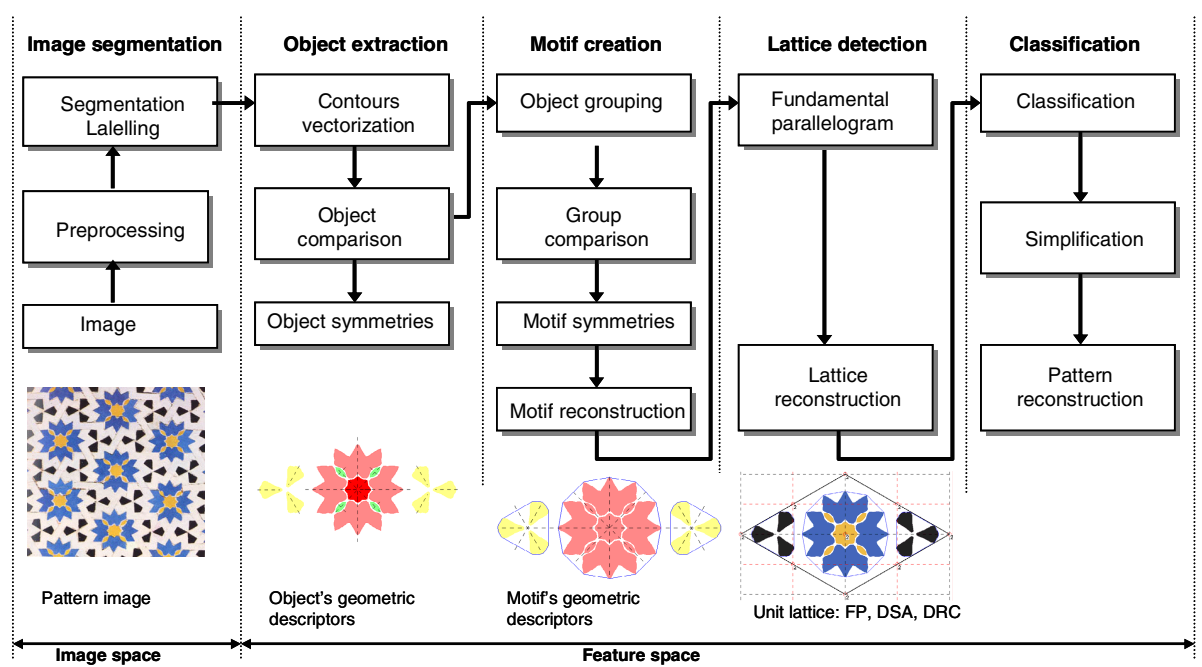

Fig. 2. Main components of the proposed computational model

than the image world. Figure 2 shows a scheme of proposed model's main components.

The underlying idea of our proposal is to reverse the typical process of producing a pattern in contexts such as ceramics, textile or graphic arts. In these contexts, the artist or graphic designer creates graphical entities or motifs using state of the art computer and acquisition tools. Then, they combine these motifs, regularly repeating them using geometric transformations, filling a flat area and producing the pattern design. Similarly, tiling can be produced including the motif inside a tile, with defined geometric form, and repeating the tiles with no gaps or overlapping. As indicated before, only a subset of geometric transformations is possible, as dictated by Symmetry Group Theory.

We propose extracting the motifs from the pattern image in the form of graphic entities, and using these entities to perform most of the work, such as computing geometric features, unit lattice or placement rules and, finally, to classify the pattern according to the symmetry group. In the process, we obtain many graphic objects, in parametric forms, such as Bezier curves or B-splines, which can be stored for later use in re-design tasks.

With this aim, we propose a computational model which has five main stages, depending on the feature space used to represent the data in each case. Below we briefly explain each stage:

Step 1. Image Segmentation. In this first stage the image is acquired and preprocessed to reduce noise and enhance its quality. Then, a colour segmentation algorithm is applied to decompose the image in homogeneous regions differentiated by colour or texture attributes. In [9] we proposed the use of CIE Luv colour spaces and clustering algorithms such as Mean-Shift or K-Means for this purpose. The output is again an image but each region (object) has been properly labelled with an index that differentiates it from the other regions and from the background. 

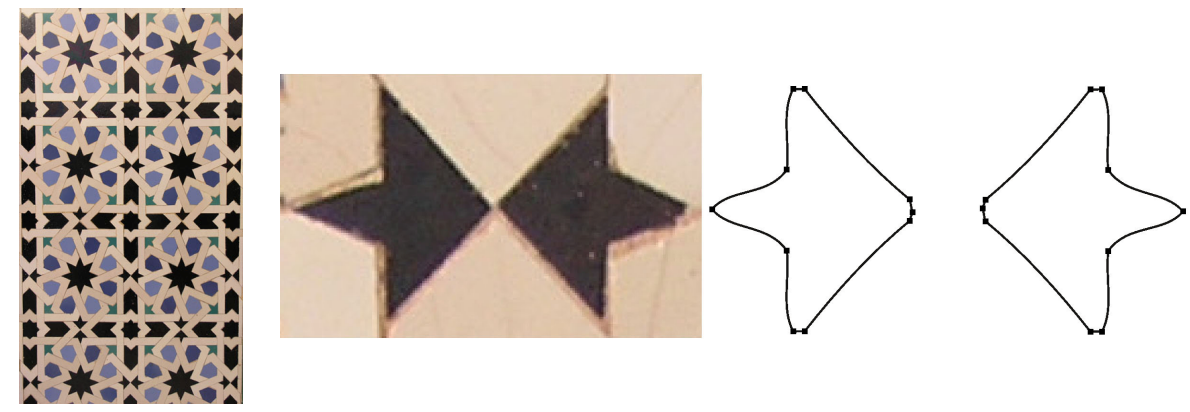

Fig. 3. Illustrative image of a historical tile design (left), detail (centre) and vectorization result (right) showing the Bezier curve nodes.

Step 2. Object Extraction. Using the labelled image as input, a vector data structure is generated. It is formed by a list of objects -which will constitute the output data-, each one of which contains a number of properties (colour, area, etc) and a list of contours (an external one and any number -zero included- of internal contours) that delimit the object's region. The contours are formed by a piece-wise sequence of Bezier curves arranged cyclically. Figure 3 (right) shows the vectorized objects found in the detail of figure 3 (centre). Within this stage there are three clearly differentiated phases:

- Contour vectorization [10]: The stage begins with a piecewise primitive approximation of the object contours using Bezier curves, by means of a two pass process: first, to obtain the border pixels sequence with a contour retrieval algorithm, and then, breaking down the point sequence into sub-sequences that are approximated by Bezier curves using a least-square method. This representation is more manageable and compact and allows scale invariance.

- Object comparison: The second sub-stage is an object comparison that attempts to obtain similar objects repeated in the image. Each set of similar objects is referred to as an 'object class'. Object comparison is limited to the external contour. To compare contours we use a more manageable feature called normalized signature, which is a representation of a fixed number of re-sampled contour points. The normalized signatures are translation and scale invariant. In [11] we describe the geometric symmetries that can be computed using signatures (reflection, rotations and shifts), and a dissimilarity measure that allows us to compare two contours. The proposed comparison method can indicate if both objects are similar (they do not exceed the similarity threshold), and the geometric transformation which links them. Figure 4 shows an example of object comparison where similar objects are drawn with the same color.

- Object symmetries: By comparing one object with itself we obtain its circular or reflected symmetry axis

Step 3. Motif Creation. A motif is a set of objects that are related by perceptual features. They are what humans first detect on visual analysis of a pattern. Even though the use of motifs to perform the PSG classification is not mandatory, we think that these entities provide us with a greater degree of abstraction and allow us to simplify the processing. In addition, they are the valuable graphic entities that users want to 
recover from a pattern to use in re-design tasks. This could be one of the most interesting contributions of this work.

Using the data structure with the list of objects as input, we can generate a list of groups or motifs, each represented by a number of related objects and a contour (minimal convex polygon that includes such objects). Figure 4 shows an example of object grouping and motif creation. This stage is similar to object extraction, in that first the working units (objects / motifs) are obtained and then compared, although the procedures used are very different:

- Object grouping: The related objects are grouped using perceptual criteria (inclusion, contact, co-circularity, co-linearity and overlapping).

- Group comparison: The comparison is done at two levels, first it is checked that the groups (motifs) contain a certain percentage of similar objects, and then the transformations relating the objects to the two motifs are compared (displacements, rotations or symmetries). The presence of a predominant transformation indicates that both motifs are formed by similar objects that are equally distributed inside the motif they belong to. Such motifs are considered to be similar, even if there are some disjoint objects, and they are sub-classified as the same class. It is very common for incomplete motifs (in the borders of the image) or motifs with gaps to appear.

- Motif symmetries: Global motif symmetries are obtained by comparing the symmetry axes and rotation centres of the objects in each motif class.
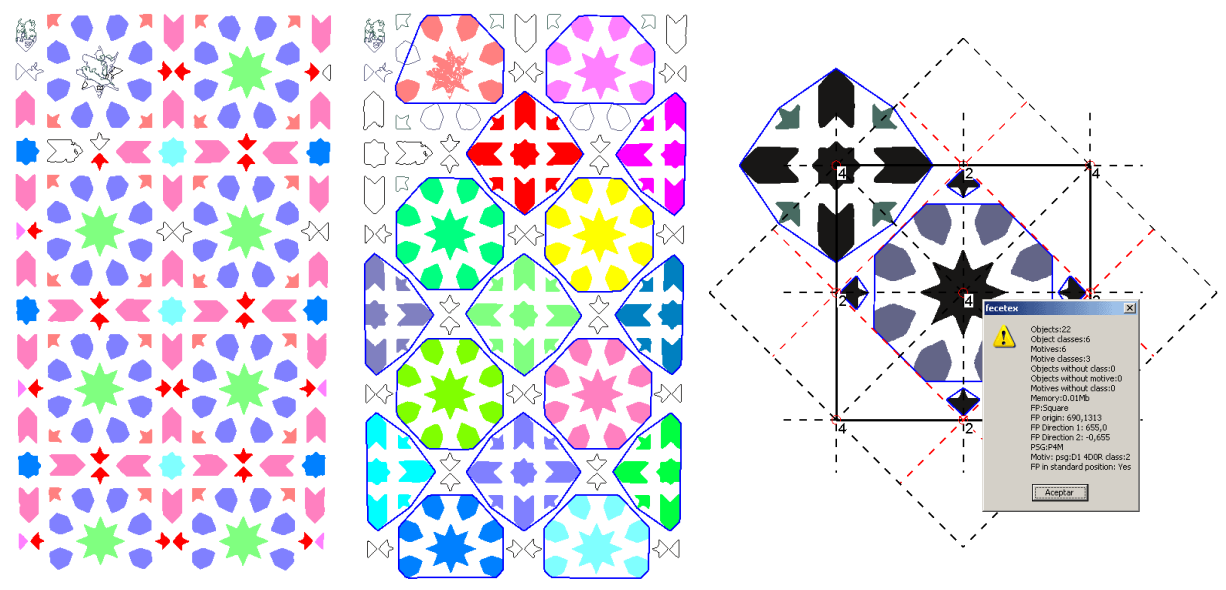

Fig. 4. Object comparison (left), motif creation (centre) and final classification (right) showing the fundamental parallelogram (black square), the symmetry axes (broken lines) and the rotation centres (circles)

- Motif reconstruction: This is the first moment at which we have enough information to start correcting errors. This correction, or reconstruction, covers two different aspects: (i) Restitution, which restores missing objects to a motif by bringing them from another motif in the same class and, (ii) unification, which unifies different objects located in the same position in their respective class. In [12] we introduced a set of rules to perform these tasks. 
Step 4. Lattice Detection. In this stage we obtain the translational symmetry of the pattern. Two operations are carried out:

- Fundamental parallelogram: There are motifs in each class related by displacements. We obtain the two unique displacement vectors that, through linear combinations, make them to coincide. These two vectors act as the basis of the vector space defined by the positions of similar motifs. As usually there are several motifs there will be several bases, so we will choose the one with an n-times area, since pattern repeatability is that of the less frequently repeated elements. Such vectors form two sides of the Fundamental Parallelogram or unit lattice: the smallest part able to generate the whole pattern by replicating and displacing.

- Lattice reconstruction [12]: While in the case of motif reconstruction we worked with loose objects, now we work with complete motifs. The repetition of fundamental parallelograms will form a mesh where all the motifs located in the same relative position within each mesh cell must be equal. If they are not, we remove some and replicate others to unify the pattern.

Step 5. Classification. In this last stage we perform the whole PSG classification. The operations in this stage are the following:

- Classification: Considering the geometry of the FP, the symmetry axes and the rotation centres, the FP is classified in accordance with Table 1.

- Simplification: The pattern is simplified, since the content of the FP and its PSG will suffice to define the whole pattern; therefore we can suppress all redundant information without decomposing any objects or motifs.

- Pattern reconstruction [12]: Once the plane symmetry group has become available, the defects can be corrected since the reflections and rotations involve the content of the motifs and even the object regions. For this purpose we check that all the objects or motifs related by symmetry axes or rotation centres are equal and following the correct orientation; otherwise, we choose the best alternatives (those which fulfil the symmetry criterion) and any incorrect ones are replaced.

Figure 4 (right) shows the analysis result for the pattern in Figure 3 (left). Only the motifs contained in the FP have been left, without dividing any of them.

\section{Experiments and Results}

To validate the proposed methodology we have successfully used 22 different tiling patterns, with repetition in two plane directions, from the mosaic collection of Pedro I's Palace in the Alcazar of Seville (Spain). Figure 5 shows an example of such mosaics. Two problems arise: the first one is related to the historical nature of these tiles which were made in the $14^{\text {th }}$ century using handcraft techniques. Consequently, there are inaccuracies in the position and finish of the mosaic tiles. The second problem is the use of a tile design technique widely extended in Islamic decoration of that period, known as "lacería" or "interlace" (Figure 5 right). They can be defined as figures, built from regular polygons or stars, developed in the form of a band that extend its sides in such a way that they alternatively cross each other, generating a composition of artistically arranged loops. 


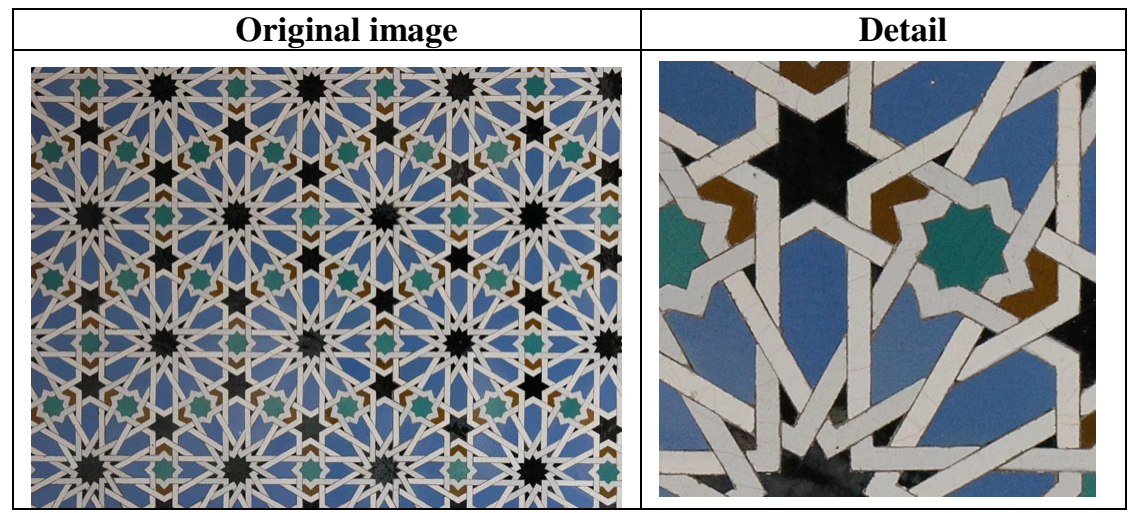

Fig. 5. Original image (left) and detail (right) from a mosaic of the Alcazar of Seville

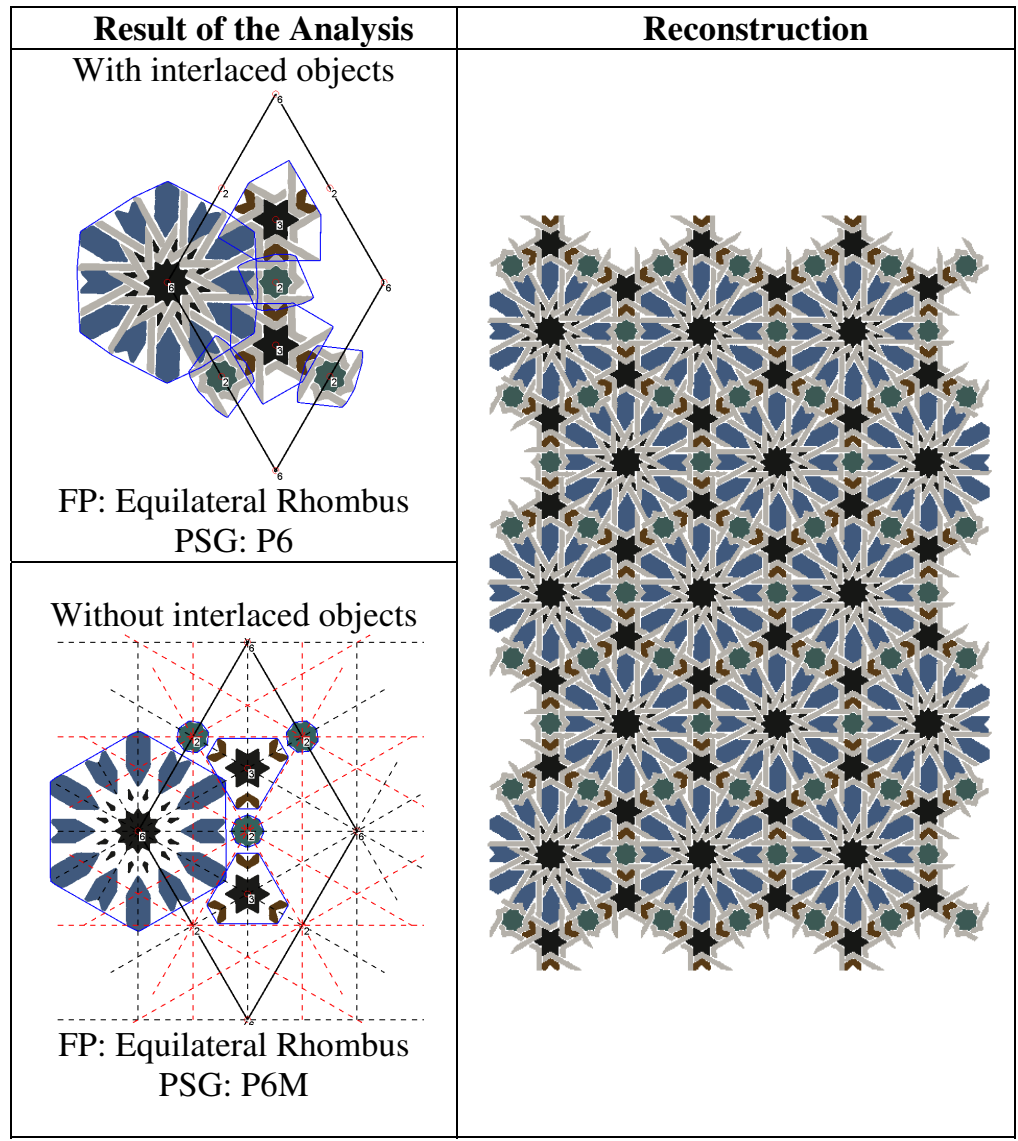

Fig. 6. Simplified result of the analysis (left) with interlaced objects (up) and without them (down), and pattern reconstruction (right) of the original mosaic in figure 5 
The treatment of the lacerias was twofold. Firstly we increased the color tolerances of the segmentation operator achieving fusion of lacerias, which were then considered as background. And secondly, we reduced the color tolerances in such a way that lacerias appeared as independent objects. Figure 6 shows the results obtained in both cases. The upper image is the simplified result of the analysis considering all the objects of the pattern, while the lower image is the result without considering the interlaced objects. The fact that the interlaced objects are always arranged circularly, without symmetry axes common to all of them, makes Plane Symmetry Group different in both cases. The image on the right shows the pattern reconstruction from the simplified analysis with interlaced objects, by repetition and displacement using the FP directions.

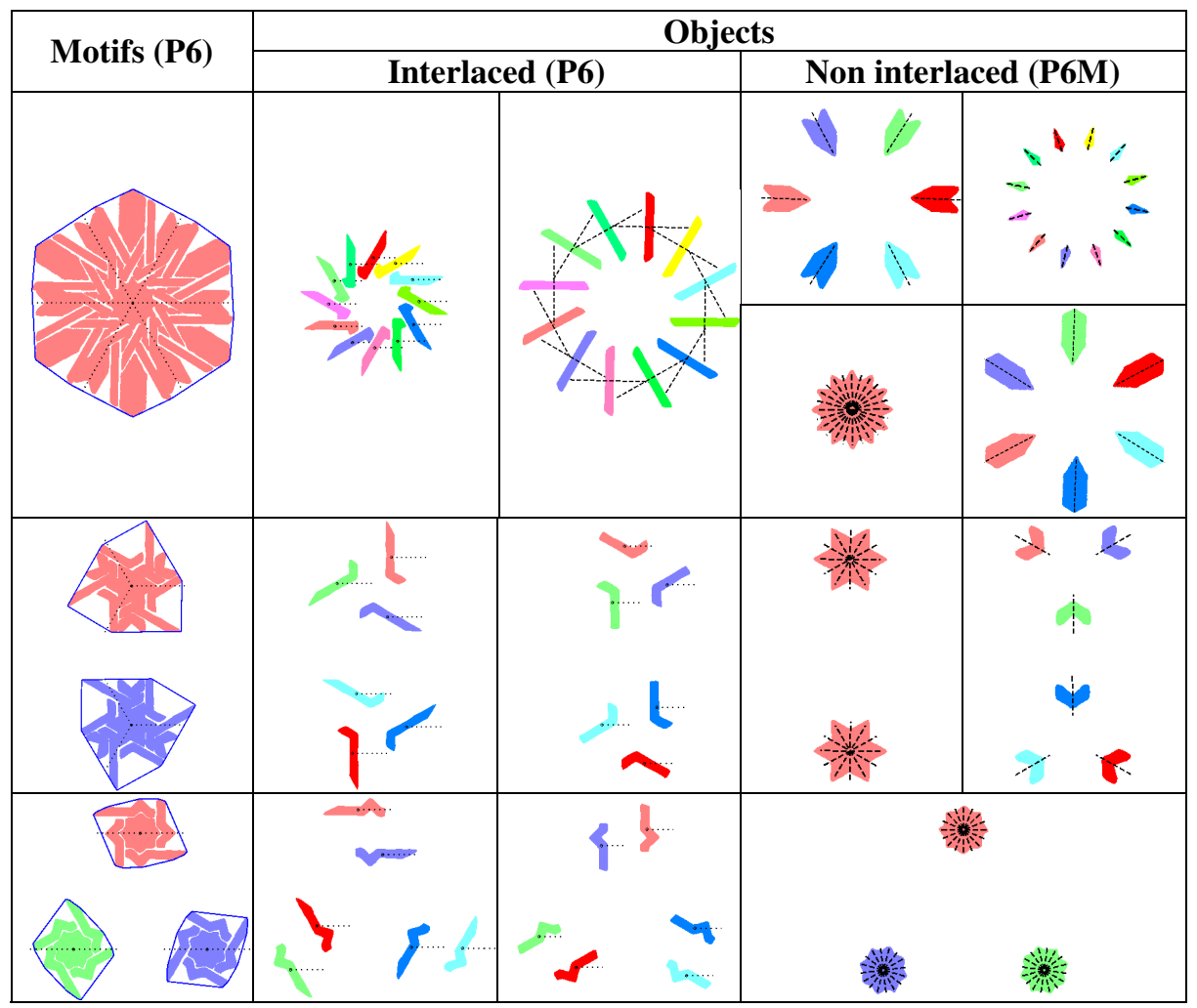

Fig. 7. Motifs and objects obtained after simplification. The interlaced objects have been separated from the rest. Symmetry axes are represented with a dashed black line and different orientations are showed by different colours.

Finally, Figure 7 shows the main motifs and their objects obtained from the analysis, including all symmetry axes. As this figure shows, the interlaced objects either do not have symmetry axes, or they are not common to all, while the other objects have symmetry axes and are common to all, so that the Plane Symmetry Groups of each type of objects, have the same PF and rotation centers, but one has symmetry axes 
(P6M) and the other does not (P6). The motifs that contain the interlaced objects have the less restrictive PSG, which is P6.

The experiments show that this computational model satisfactorily reached its objectives with most of the processed images. In some cases, the user must tune system parameters to obtain a correct classification. Figure 8 summarizes the results obtained showing the obtained PSG for the two possible classifications. Observe the high number of P4-P4M tile designs in this kind of mosaics.

Typical processing time is presented in Table 2. In the segmentation stage the time depends on the image size but, in the other stages, it depends heavily on the number of existing objects, (motifs) and their complexity, with the longest time requirement for processing the tiles with interlaced objects.

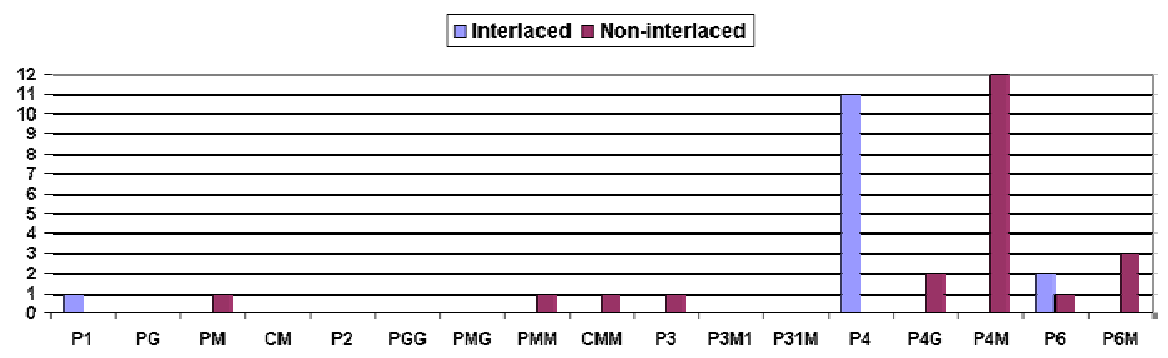

Fig. 8. Number of patterns in each PSG with and without considering interlaced objects

Table 2. Processing time for several examples on a Pentium III $450 \mathrm{MHz}$ computer

\begin{tabular}{|r|c|c|c|}
\hline & Minimum & Median & Maximum \\
\hline Time & $24{ }^{\prime \prime}$ & 1 '10” & 4 '26”' \\
\hline Image size & $3264 \times 2448$ & $3264 \times 2448$ & $3264 \times 2448$ \\
\hline Number of objects & 54 & 162 & 699 \\
\hline
\end{tabular}

\section{Conclusions}

This paper presents a computational model for analyzing periodic design patterns. The successful results obtained after analysis of tiling design patterns in the Alcazar of Seville are also reported. The main findings can be summarized as follows:

- All the tiling patterns used were successfully classified. Their structures (fundamental parallelogram and plane symmetry group) and elements (objects and motifs) were obtained in the form of graphic entities.

- The problems derived from the use of interlaced objects in most of the tiles analyzed were solved. From the data obtained, we can conclude that in the case of interlace tile design patterns it is advisable to provide the two possible classifications rather than their more generic classification (without symmetry axes).

- Compression ratios up to 1:1000, with respect to the original image in jpeg format, were obtained. The pattern structure was reduced to its fundamental parallelogram geometry and content and one object/motif per class. 
The proposed computational model behaves perfectly with all the mosaic samples used and its output data represents a meaningful and compact design description of the original pattern. This data reduction is very convenient for storing and retrieval purposes in information systems, which are a current issue in the ceramic and textile industries.

\section{Acknowledgements}

This paper has received the support of the Spanish Ministry for Science and Technology and the European Union (Project DPI2001-2713). This work has been possible thanks to the collaboration of the Real Alcázar of Seville foundation.

\section{References}

1. Schattschneider, D. The Plane Symmetry Groups: Their Recognition and Notation. The American Mathematical Monthly, vol. 85, pp. 439-450, 1978.

2. Grünbaum, B., Shephard, G.C. Tilings and Patterns, W. H. Freeman, New York, 1987.

3. Shubnikov, A. V.; Koptsik, V. A. Symmetry in Science and Art, Plenum Press, New York, 1974.

4. Atallah, J.M. "On Symmetry Detection”, IEEE Transactions on Computers, C-34, pp. 663666, 1985.

5. Dinggang, S., Ip, H.S.H, Cheung, K.T.K.; T.E. Khwang. Symmetry Detection by Generalized Complex (GC) Moments: A Close-Form Solution, IEEE Transactions on Pattern Analysis and Machine Intelligence, vol. 21, pp. 466- 476, 1999.

6. Y. Liu, Y.; Collins, R.T. and Tsin, Y. A Computational Model for Periodic Pattern Perception Based on Frieze and Wallpaper Groups, IEEE Trans. Pattern Analysis and Machine Intelligence, vol. 26, no. 3, pp. 354-371, Mar. 2004.

7. Horne C. E. Geometric Symmetry in Patterns and Tilings. Woodhead Publishing. Abington Hall, England, 1st edition (2000)

8. Valiente J.M.; Albert, F.; Carretero M. and Gomis J.M.: Structural Description of Textile and Tile Pattern Designs Using Image Processing, Proceedings of the 17th International Conference on Pattern Recognition (ICPR 2004), IEEE Computer Society Press, Cambridge (England), Aug. 2004.

9. Valiente, J.M.; Agustí, M.; Gomis, J.M. and Carretero, M.: Estudio de técnicas de segmentación de imágenes en color aplicadas al diseño textil, Proceedings of the XIII International Congress of Graphics Engineering, Badajoz (Spain), June 2001.

10. Albert, F.; Gomis, J.M.; Valor, M; Valiente, J.M. and Carretero,M. Análisis estructural de motivos decorativos en diseño textil, Proceedings of the XIII International Congress of Graphics Engineering, Badajoz (Spain), June 2001.

11. Van Otterloo, P.J. A contour-oriented approach to shape analysis, Prentice-Hall International, 1991.

12. Albert, F., Gomis, J.M.; Valiente, J.M. Reconstruction Techniques in the Image Analysis of Islamic Mosaics from the Alhambra, Proceedings of the 2004 Computer Graphics International (CGI 2004), IEEE Computer Society Press, Heraklion, Crete (Grecian), 2004.

13. Kanatane, K. Comments on Symmetry as a Continuous Feature, IEEE Transactions on Pattern Analysis and Machine Intelligence, vol. 19, no. 3, pp. 246-247, March 1997.

14. Shape Grammars. http://www.shapegrammar.org/intro.html 